\title{
Implementation of a synoptic operative note for abdominal wall hernia repair: a statewide pilot evaluating completeness and communication of intraoperative details
}

\author{
Lia D. Delaney ${ }^{1,2}$ (D) Kerry M. Lindquist ${ }^{2} \cdot$ Ryan Howard $^{2,3} \cdot$ Anne P. Ehlers $^{2,3} \cdot$ C. Ann Vitous ${ }^{2} \cdot$ Michael Englesbe $^{3,4}$. \\ Justin B. Dimick ${ }^{2,3,5}$. Dana A. Telem ${ }^{2,3,5}$
}

Received: 16 April 2021 / Accepted: 14 June 2021 / Published online: 14 July 2021

(c) The Author(s), under exclusive licence to Springer Science+Business Media, LLC, part of Springer Nature 2021

\begin{abstract}
Background Variable approaches to intraoperative communication impede our understanding of surgical decision-making and best practices. This is critical among hernia repairs, where improved outcomes are reliant on understanding the impact of different patient characteristics and surgical approaches. In this context, a hernia-specific synoptic operative note was piloted as part of an effort to create a statewide hernia registry. We aimed to understand the impact of the synoptic operative note on variable missingness and evaluate barriers and facilitators to improved intraoperative communication and note adoption. Methods In January 2020, the Michigan Surgical Quality Collaborative (MSQC) registry was expanded to capture herniaspecific intraoperative variables. A synoptic operative note for hernia repair was piloted at 8 hospitals. The primary outcome was change in hernia variable communication, measured by missingness. Using a sequential explanatory mixed-methods design, we performed semi-structured interviews with data abstractors $(n=4)$ and surgeons $(n=4)$ at 5 pilot sites to assess barriers and facilitators of implementation. Interviews were iteratively analyzed using content analysis with both deductive and inductive approaches.

Results From January to June 2020, 870 hernia repairs were performed across 8 pilot and 53 control sites. Pilot sites had significantly less missingness for all hernia-specific variables. At pilot sites, $46 \%$ of notes were fully complete in regard to hernia variables, compared to $21 \%$ at control sites $(p$ value $<0.001)$. While collection of intraoperative variables improved after synoptic note implementation, low note adoption was reported. Facilitators of improved variable collection were (1) communication with data abstractors and (2) stakeholder acknowledgment of widespread benefit, while barriers included (1) surgeon resistance to practice change, (2) EMR/technology, and (3) interruptions to communication and implementation. Conclusion This mixed-methods evaluation of a synoptic operative note implementation suggests that sustained communication, particularly with abstractors, was the most impactful intervention. Future implementation efforts may have improved effectiveness with interventions supplementary to surgeon-level direction.
\end{abstract}

Keywords Abdominal hernia repair $\cdot$ Synoptic operative note $\cdot$ Intraoperative communication $\cdot$ Variable registry

Understanding surgical decision-making and best practices in surgery often requires a detailed description of patient

Dana A. Telem

dtelem@med.umich.edu

1 University of Michigan Medical School, Ann Arbor, MI, USA

2 Center for Healthcare Outcomes and Policy, University of Michigan, Ann Arbor, MI, USA

3 Department of Surgery, University of Michigan, Ann Arbor, MI, USA and operative factors in the operative note. This is especially true for abdominal wall hernia repairs, one of the most 
commonly performed operations in the United States [1]. Similar hernias can be approached in different ways, and an inability to capture nuanced variation and its impact on outcomes limits quality improvement efforts. A major barrier to characterizing variation in intra-operative techniques which directly affects outcomes, is the lack of standardized reporting on hernia-specific elements. Operative notes are inconsistent with regard to key details such as hernia size, location, use of mesh, and mesh placement that must be understood to develop best practices and ensure appropriate care for persons with recurrent disease [2-4]. Current evidence suggests up to $67 \%$ of hernia operative notes are missing at least one of these important operative characteristics [3, 4]. Consistent operative documentation would aid in the collection of patient outcomes and long-term data, and allow for an understanding of how to best-serve patients [5].

One strategy that has been shown to be successful in standardizing the recording of data and increasing operative note completeness is the use of synoptic operative notes [2, 6-8]. A synoptic operative note is a predefined electronic template that contains an outline of procedure-specific sections for the recording of important characteristics and steps of the operation. Synoptic operative note use in other domains, including cholecystectomies, pancreatic resections, and colorectal surgeries has resulted in reduced inter-note variability and increased data completeness [6,9-12]. With evidence that synoptic operative notes improve documentation and have the potential to improve patient outcomes, the American College of Surgeons Commission on Cancer has recently announced that synoptic operative reporting will become a new required operative standard to be implemented in 2021 for several common cancer surgeries [13-15]. Despite the evidence for improvement in intraoperative documentation, this approach has not been widely adopted, particularly in fields such as hernia repair where best practices remain unknown [16].

In this context, we sought to understand the impact of a synoptic operative note on intraoperative communication of hernia-specific variables. Using a sequential explanatory mixed-methods design, we quantified operative note documentation by assessing hernia variable missingness in a statewide registry. We then performed semi-structured qualitative interviews to assess barriers and facilitators of implementation and fidelity to the intervention. We hypothesized that institutions that piloted the synoptic operative note would be associated with increased communication of key intraoperative data elements via synoptic note usage by surgeons.

\section{Methods}

This explanatory sequential mixed-methods study evaluates pilot interventions aimed at improving the intraoperative communication of hernia repairs across the State of
Michigan. The documentation and missingness of variables in a statewide registry were quantified to evaluate operative note completeness. Subsequently, semi-structured qualitative interviews were conducted with stakeholders, including both surgeons and data abstractors, at the pilot sites to determine the most impactful components of the implementation strategy and any changes in variable documentation. This study was deemed exempt by institutional review board of the University of Michigan.

\section{Study population}

The Michigan Surgical Quality Collaborative (MSQC) is a statewide quality improvement collaborative and clinical registry that comprises 70 hospitals with a strong track record of implementing quality improvement campaigns [17, 18]. The registry prospectively collects data on perioperative processes of care and 30-day clinical outcomes after operations performed at the participating hospitals. Data are reviewed and abstracted by trained data abstractors and a sampling algorithm is used to minimize selection bias [19]. In January 2020, the MSQC variable database was expanded to capture hernia-specific intraoperative variables such as hernia location, recurrence and size (Table 1). A launch meeting outlining the new variables with abstractors was conducted at each MSQC site. Abstractors from all MSQC sites had the ability to access the new variables and place data into the registry, which provided a control arm to represent the baseline impact of registry expansion on variable completeness at nonpilot sites without intervention.

\section{Pilot site intervention}

A synoptic operative note for hernia repairs was designed to improve and standardize statewide communication of key intraoperative data (Supplemental File 1). In January 2020, 8 diverse sites in the MSQC were selected with respect to location, practice type, and practice size and agreed to pilot a synoptic operative report for ventral and incisional hernia repairs. Surgeon champions were identified from each site to introduce the synoptic operative note at their institution. Surgeons were given an opportunity to provide iterative feedback on the operative note content and layout prior to its launch. Various formats of the note (electronic and paper) were distributed to the pilot sites to maximize usability and overcome differences in institutional documentation methods and electronic medical record (EMR) systems. Supplementing note implementation at pilot sites were longitudinal engagement efforts aimed at multilevel stakeholders including meetings with data abstractors regarding the impact of poor intraoperative communication as well as education sessions detailing how registry variables correlate to clinical data and operative steps. 
Table 1 Hernia-specific variables added to the MSQC variable registry in January 2020

\begin{tabular}{ll}
\hline Hernia variables & Data element details \\
\hline Hernia location & Epigastric, umbilical, infraumbilical, suprapubic, no midline component \\
Initial or recurrent & Did the patient have a previous hernia repair? \\
Hernia width & Width in cm \\
Hernia length & Length in cm \\
Mesh placement & Was mesh used? \\
Mesh width & Width in cm \\
Mesh length & Length in cm \\
Type of mesh & Synthetic nonabsorbable, synthetic absorbable, biosynthetic, biologic, other \\
Mesh brand & Brand of mesh (e.g., Bard, Medtronic, Ethicon, etc.) \\
Mesh location & Onlay, inlay, sublay \\
Sublay location & Retrorectus, preperitoneal, intraperitoneal \\
Mesh fixation & Suture, adhesive, absorbable tacks, non-absorbable tacks, self-fixating, other/multiple \\
Myofascial release & Was myofascial release performed? \\
Myofascial release type & Anterior versus posterior \\
\hline
\end{tabular}

\section{Intraoperative communication}

The primary outcome was communication and collection of MSQC hernia-specific intraoperative variables. Variable missingness among operative notes was compared between 8 pilot and 53 control sites for the following hernia-specific variables: location, recurrence, hernia length, hernia width, use of mesh, mesh type, and component separation. The number of fully completed operative notes and operative notes missing all specified hernia variables was also compared between pilot and control sites.

\section{Qualitative data collection}

To further understand the effect of this intervention on surgeon practice as well as barriers and facilitators to its adoption, implementation outcomes were assessed 6 months after the pilot launch of the synoptic operative note. Invitations to complete an interview with a study team member were sent via email to 8 surgeons and 8 abstractors from the pilot sites. Qualitative interviews with data abstractors and surgeons were completed from July to September 2020 via telephone, by one investigator (L.D.D) with previous experience conducting qualitative interviews. Interviews were semistructured, audio-recorded and lasted 15-20 min in length. Interviews continued until data saturation was achieved, determined when new themes emerged infrequently, and code definitions remained stable [20]. All interviews were deidentified prior to transcription.

\section{Interview analysis}

The interview data was iteratively analyzed using content analysis and incorporated both deductive and inductive approaches. The deductive components utilized the Theoretical Domains Framework to generate an approach to assessing implementation outcomes, with the assumption that each pilot site had adopted the synoptic operative note [21]. Specifically, degrees of feasibility, acceptability, and effectiveness were targeted to determine barriers and facilitators to the intervention, with queries that were mapped to each domain [21]. Interview content was shifted when note uptake was realized to be low, and an inductive analysis was used to allow for the identification of domains not captured by the framework. The initial codebook was created by having two team members (L.D.D., K.M.L.) independently review the transcripts and come together to collate ideas, mapping them to a coding schema. Discrepancies were discussed to consensus and codes were refined to develop a final codebook, with input from additional team members with expertise in implementation outcomes and qualitative data (D.A.T., C.A.V.). Two team members (L.D.D., K.M.L.) independently coded the transcripts using the final codebook using NVivo software (Version 12.6.0) and met to ensure consensus after each transcript [20]. After the data were fully coded, all team members reviewed the code summary to reach consensus on the most salient themes.

\section{Results}

\section{Quantitative results}

\section{Patient and operative characteristics}

From January 1 to June 12, 2020, 870 eligible hernia repairs were performed, of which 157 (18\%) were performed at 8 pilot sites and $713(82 \%)$ were performed at 53 control sites. 
Among the 870 patients, mean (SD) age was 55 (15) years old and 461 (53\%) patients were male, with no significant difference in age or sex between pilot and control sites.

\section{Intraoperative communication}

Hernia repairs performed at pilot sites had significantly less missingness for all hernia-specific variables (Table 2). The variables with the largest difference in missingness were myofascial release (4\% at pilot sites vs $35 \%$ at control sites; $p$ value $<0.001)$, hernia width ( $49 \%$ at pilot sites vs $68 \%$ at control sites; $p$ value $<0.001$ ), and type of mesh when used ( $2 \%$ at pilot sites vs $21 \%$ at control sites $p$ value $<0.001$ ). Of the 157 cases performed at pilot sites, 95 (46\%) were fully complete and $0(0 \%)$ had all variables missing. Of the 713 cases performed at control sites, $228(21 \%)$ were fully complete $(p$ value $<0.001)$ and $20(3 \%)$ had all variables missing ( $p$ value $<0.001$ ). These results informed the qualitative analysis, focused on pilot sites to understand the aspects of the intervention that were most impactful, and to recognize potential barriers to scaling the intervention in the future.

\section{Qualitative results}

\section{Fidelity to the intervention}

Five out of 8 pilot sites had a representative stakeholder complete an interview. Interviews were conducted with 4 surgeons and 4 data abstractors. Only one site reported adoption of the synoptic operative note. An interview script was designed to assess implementation outcomes, with the assumption that each pilot site had adopted the synoptic operative note. However, when surgeon utilization of the synoptic operative note was realized to be low, interview content was shifted. Interviews instead elicited potential explanations for the decreased variable missingness at pilot sites as well as surgeon and data abstractor perspectives on barriers and facilitators to synoptic operative note usage. One additional site that did not complete an interview, reported via email that they had just begun piloting the note and requested additional resources to aid in EMR integration.

\section{Qualitative content analysis}

Several facilitators and barriers emerged as impactful in intervention efforts to improve variable collection. Facilitators included (1) engagement and communication with data abstractors, and (2) stakeholder acknowledgment of widespread benefit. Barriers included (1) surgeon resistance to practice change, (2) EMR/technology, and (3) interruptions to communication and implementation.

\section{Facilitators to improved variable collection}

\section{Engagement and communication with data abstractors}

Consistent engagement and communication with data abstracters emerged as the most impactful intervention to improve variable collection. The responses of surgeons and abstractors at pilot sites varied in whether surgeon notes had improved in quality or changed to include new hernia variables, but all abstractors reported a personal change in data abstraction techniques. Data abstractors at pilot sites reported often searching outside of the operative note in other locations of the patients' charts to find the key variables. Changes to abstraction methods was attributed to various abstractor-directed interactions from the implementation team, such as institution-level meeting attendance by project leaders to launch and explain the initiative, consistent correspondence and iterative feedback via email surveys, and surgeon-led education sessions designed to outline the importance of the data collection and clinical correlations of the variables.

"I used to go through the hernia operative note to see if there were any drains, issues, bowel-related complications, or any of that. Now, I go through with a finetooth comb ... to find all the correct stuff and to make sure that I am understanding the procedure right, reading the note better, and filling out the [information]
Table 2 Missingness rates of hernia-specific variables at pilot and control sites

\begin{tabular}{lllr}
\hline Hernia variables & $\begin{array}{l}\text { Missingness at pilot sites } \\
(n=8) \%\end{array}$ & $\begin{array}{l}\text { Missingness at control sites } \\
(n=53) \%\end{array}$ & $P$ value \\
\hline Hernia location & 2 & 10 & 0.001 \\
Recurrent hernia & 0 & 7 & 0.001 \\
Hernia length in cm & 29 & 38 & 0.032 \\
Hernia width in cm & 49 & 68 & $<0.001$ \\
Mesh used: Yes or No & 1 & 4 & 0.056 \\
Type of mesh (when used) & 2 & 21 & $<0.001$ \\
Myofascial release & 4 & 35 & $<0.001$ \\
\hline
\end{tabular}


correctly... I spend more time making sure that the questions are answered to the best of my knowledge." [Abstractor 4]

"I have been using all of the information that was given to us...there was a doctor that gave us tips during an education session. I went through and printed out some information, so that when I am abstracting, I am gathering as much as I can.” [Abstractor 2]

\section{Stakeholder acknowledgment of widespread benefit}

Many surgeons and abstractors indicated support for the initiative and an understanding of the widespread benefit of more complete operative notes and the potential for improved hernia care overall. Both abstractors and surgeons referenced the variability in operative note characteristics and completeness among surgeons. Both groups also recognized the advantages of operative note standardization, including improved record-keeping and research pursuits. Abstractors reported that the effort of including all of the variables did not cost them extra time on average; however, the potential time added by writing a different or additional note remained a concern for surgeons. Although not all surgeons adopted the synoptic note, many reported personal efforts to improve their own templated operative notes and include key variables in light of education efforts surrounding the synoptic operative note intervention. Additionally, there was several requests from surgeons for another template of the synoptic operative note, reflecting interest in working towards the intervention, and a potential gap in surgeon engagement from the implementation team.

“That's why I think this operative note is such a great idea... I am such a proponent for gathering this data. I think it is so valuable and I see the value of the variable registry, so I am always on board." [Abstractor 3]

"I would love to get [the note] here if we could-I think it would be helpful overall. Especially for the whole state and for being able to do future research on best practices in hernia repairs and approaches. I would love to keep moving forward with it." [Surgeon 3]

\section{Barriers to improved variable collection}

\section{Surgeon resistance to adoption of new practices}

Overall, there was an acknowledgment that an understanding of the global benefit of a synoptic operative note may not be sufficient to incentivize surgeon practice change or institutional support. Resistance to the adoption of new practices emerged as a barrier to both usage of the synoptic operative note and general operative note completeness. Both surgeons and abstractors acknowledged that surgeon-level change has been a challenge in past quality improvement interventions and would require time to overcome. This was especially highlighted among institutions whose current operative note records were not already templated and were instead completed through dictations and paper charts. Surgeon length of practice also emerged as a contributor to practice change resistance, with the consensus being that the longer a surgeon had been in practice, the less likely they were to change. However, optimism for change was consistently acknowledged by both the abstractors and surgeons. During interviews, data abstractors and surgeons advised to continue consistent communication with surgeon champions regarding priority variables and their importance while also increasing the ease of note utilization through efforts to integrate the new note with standard operative notes.

"The only concern I have is about feasibility moving forward. I think there is a lack of ability to adapt to new things. Teaching an old dog new tricks... you know?" [Surgeon 4]

"The surgeons are so set in their ways and it's hard for them to adapt to new things... maybe it is just more groundwork you have to lay ahead of time and then they can adjust, adapt, and work on it. Not an overwhelming enthusiasm in the beginning, that's for sure." [Abstractor 3]

\section{EMR and technology}

Every pilot site attributed a component of the delay in synoptic note implementation to technology barriers or limitations of their institutional EMR. This included a lack of surgeon time or knowledge to integrate the template into differing EMR systems, challenges coordinating with and incentivizing local information technology (IT) departments to assist with the implementation, and limited administrative support at the institutions.

"It hasn't really gotten off the ground here because it is not integrated into our EMR, which is a pretty major barrier and without it, there was not much of an incentive to do two notes." [Abstractor 3]

"When I went to IT, they said they did not know how to incorporate it and it fell apart. I don't know if there is another system or if there is a way that I can get my IT people on board in an easy fashion. I think that is what I am going to need to go forward." [Surgeon 3] 


\section{Interruptions to communication and implementation}

The synoptic operative note was promoted to varying levels at each institution. Most surgeons had an initial meeting with their team where they explained the idea with variable receptiveness to adoption from their surgeon teams. The majority of surgeons reported conducting this meeting later in the pilot period, at which time they could not provide an answer to all the questions their team members asked. Of the surgeons contacted, there were 3 who requested additional resources such as the synoptic operative note template or instructions on how to integrate the note into the EMR, including 1 surgeon who did not complete an interview. Abstractors noted that they had a role in the initial implementation of the synoptic operative note launch in internal meetings with surgeons, but also expressed that the primary responsibility of promoting clinical use should be with the surgeons. This was attributed to the surgeons' experience and qualifications that abstractors believed would be respected by colleagues and would strengthen the endorsement.

"I literally have not been able to [promote the note with surgeons] at all. I am not even sure if [the surgeon champion] was able to disseminate that information at the general surgeons meeting. Because of COVID-19, everything got shut down and there were no big meetings going on, so I don't know what the rest of the surgeons know. [Abstractor 2]

"I see the role of [note promotion] to be the surgeon champion because they listen to him. He has the experience and will use that. He is very good at adapting. He will say, "Ok, I have done this, it's not that hard, and this is all you have to add for the project." It is much easier for the surgeon champion to present [the operative note] to [the surgeons]. I might give him the basics, but his experience using it and his knowledge of how it can be used is much more helpful." [Abstractor 3]

Disruptions related to COVID-19 were consistently cited as a barrier in note utilization and promotion. Relevant consequences included a decrease in the number of hernia surgeries performed, data abstractors who were registered nurses being redeployed to work clinically, and an overall decrease in internal communication. The transition to remote communication and off-site administrative work in addition to the number of canceled meetings hindered communication between surgeons and abstractors and acted as a main barrier to effective intra-institution communication. Consistent multidisciplinary communication within the institutions at such meetings was reported to be a key facilitator of previous institutional interventions directed at practice change.

"I have not had a meeting with my surgeons because we are not allowed to have any right now. I am hoping to do a WebEx to check-in with them about it, but I have not yet. [Abstractor 4]

"If the surgeons are on board, that would be the easiest! It is similar to other new initiatives at first-trying to get them to adapt to how they record, and for us to adapt to what we were collecting and how we were finding information... it takes time. And COVID-19 came and messed things up, too." [Abstractor 1]

\section{Discussion}

While communication and data collection of intraoperative variables had improved 6 months after implementation of a synoptic operative note, low adoption of the note was reported. Despite the association between synoptic operative note intervention at pilot sites and decreased variable missingness, qualitative interviews disproved the assumption that synoptic note adoption by surgeons was solely responsible for the change, instead revealing that efforts by data abstractors to adapt current practices and expand variable collection drove the success of variable completeness at pilot sites. These results represent the first mixed-methods evaluation of the implementation of a synoptic operative note with input from multiple levels of stakeholders in the intervention and suggest that sustained communication, particularly with abstractors, was more impactful to improved variable collection than the synoptic operative note itself. The granularity of these results will inform the direction of future implementation efforts to include a multidisciplinary approach and could have only been obtained from a mixed-methods study with qualitative feedback on the implementation.

Up to two-thirds of hernia operative notes are missing a key clinical detail of the operation, and despite synoptic operative notes improving documentation in other domains, they have yet to be implemented among hernia surgeries [4, 6-8]. In our multi-institution implementation of a synoptic operative note for hernia repairs, a level of surgeon resistance to altering current practices was reported. This is consistent with previous qualitative findings that discuss surgeon resistance as a barrier to note implementation across multiple institutions [11]. However, among other implementation efforts at single institutions, synoptic operative note usage has resulted in improved completeness of operative data without compromising surgeon satisfaction in regard to operative reporting style [10,22]. This may be a product of the scale of the intervention. The targeting of 
small engaged groups of surgeons at single institutions may results in greater success by bypassing both the barrier of the project "pitch" to non-colleagues as well as inherent communication challenges of cross-institutional work [10, 22]. However, despite note adoption not occurring at every institution in our pilot intervention, there was acknowledgment of the widespread benefit of synoptic notes reported by all participants. Surgeons specifically reported efforts to improve their own operative note quality in light of the intervention regardless of whether they adopted the note. Several surgeons requested that the synoptic operative note was re-sent to them, endorsing an interest in continuing to trial it at their institution. The potential benefit to future surgeons performing reoperations and research efforts to identify best practices was also acknowledged by most surgeons and abstractors. This discordance in surgeon response and actions reflect the known challenges of quality improvement efforts and the existence of a "practice gap" between evidence-based conclusions and clinical practice change [23, 24].

The success in improved documentation and decreased variable missingness, as evidenced quantitatively, was revealed to be more than only surgeon behavior change and largely a product of commitment from data abstractors. While the variables are required to be documented originally, the commitment of other stakeholders is equally as important. Abstractors reported looking outside of the operative note for clinical variables in other locations in the patient chart at times. This suggests improved variable collection may require efforts to engage stakeholders beyond the level of the surgeon. This intervention was aided by its conduction within an existing collaborative hospital network, the Michigan Surgical Quality Collaborative, which has implemented prior quality improvement initiatives through the pre-existing communication framework. However, most institutions do not have the workflow or staff resource of trained operative note data abstracters. As such, aspects of the intervention may have to be adapted for implementation in settings that lack a collaborative hospital network. Thus, in order for the improved intraoperative documentation demonstrated in this pilot to be scalable, interventions supplementary to surgeon-level direction may be necessary. The most impactful intervention in our study was consistent and longitudinal communication with abstractors which could be directed to other onsite stakeholders or project sponsors in lieu of abstractors. There is potential for operating room nurses to absorb this responsibility, as they are commonly recruited to record the key variables themselves (implant records, defect sizes, etc.) and could be empowered to prompt participating surgeons to document them after cases.

A major barrier to note implementation at pilot sites was surgeon resistance to practice change. This supports findings by Kanters et al. that limited utilization of synoptic operative notes in colorectal surgery was due surgeon adherence and stakeholder engagement [25]. At pilot sites, the COVID-19 pandemic caused communication challenges and interruptions to elective surgeries that stalled the flow of information within departments and thus the local expansion of the synoptic note usage. This also likely affected surgeon engagement from the implementation team, as surgeons often expressed having not focused on the intervention until the interview and requested to receive another copy of the synoptic note. Improved engagement and communication with the surgeon teams could improve fidelity in the future. Additionally, 6 months is a short follow-up time for an intervention focused on practice change, and with continued efforts to reinforce the note adoption with surgeon teams, improved success may be possible. However, it is well-documented that surgeon practice change, regardless of individual motivation, is contingent on a supportive and resource-rich environment [26]. This underscores the impact of surgeon-level barriers that emerged from our interviews, such administrative and technology-related challenges, despite the acknowledged widespread benefit of synoptic note usage. Future efforts to increase note usage via surgeon engagement should be focused on mitigating institutional barriers that amplify resistance, such as medical record integration and IT challenges. With administrative support, IT departments could be contacted directly in order to streamline synoptic note incorporation with local EMRs and ease barriers to uptake. Alternatively, the distribution of educational materials regarding user-driven implementation of the note into EMR systems could empower surgeons to adapt their notes without relying on IT departments.

Despite the aforementioned barriers, both abstractors and surgeons were confident that the majority of challenges could be overcome with time and additional focused efforts, revealing an overall positive perception of the feasibility of the project. The success in decreased missingness at pilot sites documented here was after just 6 months of the intervention and during the spring of 2020, through which the COVID-19 pandemic affected healthcare delivery in every domain. In the future, if institutions are able to support surgeon willingness to improve intraoperative documentation through the synoptic operative note integration into the EMR, and engagement efforts with data abstractors continue, there is an opportunity for further improvement in variable collection.

\section{Limitations}

There are several limitations to the current study, including the sample size of the qualitative data gathered. Of the 8 pilot sites, 5 were represented with interviews, and, as with all qualitative studies, the purpose of conducting stakeholder interviews is not to be widely generalizable but to provide 
insight representative of the participants that extends quantitative conclusions. The 2 pilot sites without email or interview contact were likely less engaged in the implementation process and may contribute alternative barriers to implementation, not captured here. Furthermore, abstractors and surgeons at control sites were not interviewed, which may have provided further insight as to barriers to variable communication and reasons for variable missingness. However, the scope of this study was focused on the impact of a synoptic operative note, and thus interviews were limited to those who were introduced to the intervention.

\section{Conclusion}

This study describes the evaluation of a statewide synoptic operative note for hernia repairs and the change in intraoperative communication and note completeness after intervention. Qualitative interviews revealed that efforts by data abstractors to adapt and expand variable abstraction from operative notes were an integral component of the successful improvement in variable collection. Communication and continued engagement with data abstractors was found to be the most impactful intervention to improve intraoperative variable missingness, suggesting that improved variable collection requires efforts to engage stakeholders beyond the level of the surgeon, and that similar roles could be leveraged in future efforts to optimize surgeon behavior. Results from this study will inform a larger scale quality improvement effort to improve variable collection and operative note documentation at a statewide level.

Supplementary Information The online version contains supplementary material available at https://doi.org/10.1007/s00464-021-08614-8.

Funding Lia Delaney received the 2020 Medical Student Research Grant from the Society of American and Gastrointestinal Endoscopic Surgeons (SAGES) for this work.

\section{Declarations}

Disclosures Ryan Howard receives unrelated funding from the Blue Cross Blue Shield of Michigan Foundation and the National Institute of Diabetes and Digestive and Kidney Diseases (5T32DK10874005). Michael Englesbe receives unrelated funding from the Michigan Department of Health and Human Services, and the National Institute on Drug Abuse. Justin Dimick receives Grant funding from the National Institute of Diabetes and Digestive and Kidney Diseases, and is a cofounder of ArborMetrix, Inc. Dana Telem receives funding from AHRQ K08HS025778-01A1 and receives consulting fees from Medtronic. Anne P. Ehlers, Kerry Lindquist, Lia D. Delaney, and C. Ann Vitous have no conflicts of interest or financial ties to disclose. The Michigan Surgical Quality Collaborative is funded as part of the Blue Cross Blue Shield of Michigan Value Partnership program. The content of this study is solely the responsibility of the authors and does not necessarily reflect the official views of Blue Cross Blue Shield of Michigan. No funder or sponsor had any role in the design and conduct of the study; collection, management, analysis, and interpreta- tion of the data; preparation, review, or approval of the manuscript; or decision to submit the manuscript for publication.

\section{References}

1. Poulose BK, Shelton J, Phillips S, Moore D, Nealon W, Penson D, Beck W, Holzman MD (2012) Epidemiology and cost of ventral hernia repair: making the case for hernia research. Hernia 16:179-183. https://doi.org/10.1007/s10029-011-0879-9

2. Edhemovic I, Temple WJ, De Gara CJ, Stuart GCE (2004) The computer synoptic operative report-a leap forward in the science of surgery. Ann Surg Oncol 11:941-947. https://doi.org/10.1245/ ASO.2004.12.045

3. Porterfield JR, Altom LK, Graham LA, Gray SH, Urist MM, Hawn MT (2011) Descriptive operative reports: teaching, learning, and milestones to safe surgery. J Surg Educ. https://doi.org/ 10.1016/j.jsurg.2011.06.015

4. Ma GW, Pooni A, Forbes SS, Eskicioglu C, Pearsall E, Brenneman FD, McLeod RS (2013) Quality of inguinal hernia operative reports: room for improvement. Can J Surg 56:393-397. https:// doi.org/10.1503/cjs.017412

5. Lyons TF, Payne BC (1974) The relationship of physicians' medical recording performance to their medical care performance. Med Care 12:714-720

6. Shaikh FA, Alvi AR, Shahzad N, Gala T, Murtaza G (2019) Template operative note, a better documentation. J Pak Med Assoc 69:409-411

7. Nicopoullos JDM, Karrar S, Gour A, Panter K (2003) Significant improvement in quality of caesarean section documentation with dedicated operative proforma-completion of the audit cycle. J Obstet Gynaecol (Lahore) 23:381-386. https://doi.org/10.1080/ 0144361031000119547

8. Barritt AW, Clark L, Cohen AM, Hosangadi-Jayedev N, Gibb PA (2010) Improving the quality of procedure-specific operation reports in orthopaedic surgery. Ann R Coll Surg Engl 92:159-162. https://doi.org/10.1308/003588410X12518836439245

9. Maniar RL, Hochman DJ, Wirtzfeld DA, McKay AM, Yaffe CS, Yip B, Silverman R, Park J (2014) Documentation of quality of care data for colon cancer surgery: comparison of synoptic and dictated operative reports. Ann Surg Oncol 21:3592-3597. https:// doi.org/10.1245/s10434-014-3741-3

10. Park J, Pillarisetty VG, Brennan MF, Jarnagin WR, D’Angelica MI, Dematteo RP, Coit DG, Janakos M, Allen PJ (2010) Electronic synoptic operative reporting: assessing the reliability and completeness of synoptic reports for pancreatic resection. J Am Coll Surg 211:308-315. https://doi.org/10.1016/j.jamcollsurg. 2010.05.008

11. Deal SB, D’Angelica MI, Hawkins WG, Pucci M, Ujiki M, Brunt LM, Wexner S, Alseidi AA (2018) Synoptic operative reporting for laparoscopic cholecystectomy and pancreaticoduodenectomy: a multi institutional pilot study evaluating completeness and surgeon perceptions. Am J Surg 216:935-940. https://doi.org/10. 1016/j.amjsurg.2018.06.008

12. Bidwell SS, Merrell SB, Poles G, Morris AM (2020) Implementation of a synoptic operative report for rectal cancer: a mixedmethods study. Dis Colon Rectum. https://doi.org/10.1097/DCR. 0000000000001518

13. American College of Surgeons, Commission on Cancer (2020). Optimal resources for cancer care: 2020 standards. Comm Cancer Am Coll Surg. Available at: https://www.facs.org/quality-progr ams/cancer/coc/standards/2020. Accessed 5 Feb 2021 
14. Zhao B, Blair SL, Katz MHG, Lowy AM, Kelly KJ (2020) Adherence with operative standards in the treatment of gastric cancer in the United States. Gastric Cancer 23:550-560. https://doi.org/10. 1007/s10120-019-01028-5

15. American College of Surgeons (2020). ACS launches Cancer Surgery Standards Program to improve quality of surgical cancer care Available at: https://www.facs.org/media/press-releases/ 2020/cssp-launch-072220. Accessed 5 Feb 2021

16. Melton GB, Burkart NE, Frey NG, Chipman JG, Rothenberger DA, Vickers SM (2014) Operative report teaching and synoptic operative reports: a national survey of surgical program directors. J Am Coll Surg 218:113-118. https://doi.org/10.1016/j.jamco llsurg.2013.09.004

17. Campbell DA, Englesbe MJ, Kubus JJ, Phillips LRS, Shanley CJ, Velanovich V, Lloyd LR, Hutton MC, Arneson WA, Share DA (2010) Accelerating the pace of surgical quality improvement: the power of hospital collaboration. Arch Surg 145:985-991. https:// doi.org/10.1001/archsurg.2010.220

18. Campbell DA, Kubus JJ, Henke PK, Hutton M, Englesbe MJ (2009) The michigan surgical quality collaborative: a legacy of Shukri Khuri. Am J Surg. https://doi.org/10.1016/j.amjsurg.2009. 08.002

19. Nikolian VC, Regenbogen SE (2019) Statewide clinic registries: the michigan surgical quality collaborative. Clin Colon Rectal Surg 32:16-24. https://doi.org/10.1055/s-0038-1673350

20. Varpio L, Ajjawi R, Monrouxe LV, O’Brien BC, Rees CE (2017) Shedding the cobra effect: Problematising thematic emergence, triangulation, saturation and member checking. Med Educ 51:4050. https://doi.org/10.1111/medu.13124
21. Telem DA, Dimick J, Skolarus TA (2018) Dissecting surgeon behavior: leveraging the theoretical domains framework to facilitate evidence-based surgical practice. Ann Surg 267:432-434. https://doi.org/10.1097/SLA.0000000000002506

22. Harvey A, Zhang H, Nixon J, Brown CJ (2007) Comparison of data extraction from standardized versus traditional narrative operative reports for database-related research and quality control. Surgery 141:708-714. https://doi.org/10.1016/j.surg.2007.01.022

23. Morris ZS, Wooding S, Grant J (2011) The answer is 17 years, what is the question: understanding time lags in translational research. J R Soc Med 104:510-520. https://doi.org/10.1258/jrsm. 2011.110180

24. Meshikhes AWN (2015) Evidence-based surgery: the obstacles and solutions. Int J Surg 18:159-162. https://doi.org/10.1016/j. ijsu.2015.04.071

25. Kanters AE, Vu JV, Schuman AD, Van Wieren I, Duby A, Hardiman KM, Hendren SK (2019) Completeness of operative reports for rectal cancer surgery. Am J Surg. https://doi.org/10.1016/j. amjsurg.2019.09.036

26. Arroyo NA, Gessert T, Hitchcock M, Tao M, Smith CD, Greenberg C, Fernandes-Taylor S, Francis DO (2021) What promotes surgeon practice change? A scoping review of innovation adoption in surgical practice. Ann Surg 273(3):474-482. https://doi.org/10. 1097/SLA.0000000000004355

Publisher's Note Springer Nature remains neutral with regard to jurisdictional claims in published maps and institutional affiliations. 